\title{
TENDÊNCIAS E DESAFIOS DO PREPARO CIRÚRGICO DAS MÃOS
}

\author{
Trends and challenges of surgical hand preparation \\ Tendencias y desafíos em la preparación quirúrgica de las manos
}

Julia Yaeko Kawagoe ${ }^{1}$

RESUMO: Objetivo: Descrever tendências e desafios no preparo cirúrgico das mãos. Método: Revisão de literatura narrativa, consulta aos manuais internacionais e nacionais atuais, além de consulta à Cochrane Database of Systematic Reviews, e ao portal BVS, à base de dados LILACS, IBECS, MEDLINE, Nursing Reference Center e SciELO, utilizando os descritores: Desinfecção das Mãos, Salas Cirúrgicas; Hand Disinfection, Operating Rooms; Desinfección de las Manos, Quirófanos e conectores booleanos AND/OR no período entre 2006 e 2016. Resultados: Foram consultados cinco manuais sobre preparo cirúrgico das mãos; duas normatizações de avaliação de produtos parapreparo cirúrgico das mãos. Dos 22 artigos identificados na busca, foram selecionados sete: quatro revisões de literatura, sendo três revisões sistemáticas; e três estudos avaliando o custo e a sustentabilidade ecológica. Conclusão: Nas últimas décadas, houve grandes mudanças quanto ao tipo de produto antisséptico — favorecendo o uso de preparação alcoólica (PA), sem o uso de água e escova, representando custo-efetividade e sustentabilidade ecológica quando comparada aos procedimentos tradicionais como a degermação cirúrgica das mãos com Polivinilpirrolidona Iodo (PVP-I) ou Gluconato de Clorexedina (CHG). Para incorporar melhores práticas baseadas em evidências científicas, deve-se adotar abordagem programática, implementar políticas e programas que regem os processos e produtos utilizados, bem como o controle dessecumprimento. Palavras-chave: Desinfecção de mãos. Salas cirúrgicas. Higiene das mãos.

ABSTRACT: Objective: To describe trends and challenges of surgical hand preparation. Method: Narrative literature review, consulting the current international and national guidelines and searching the following databases: the Cochrane Systematic Reviews and the VHL portal (Latin American and Caribbean Center on Health Sciences Information), LILACS, IBECS, MEDLINE, Nursing Reference Center, and SciELO, using the keywords: Desinfecção das Mãos, Salas Cirúrgicas; Hand Disinfection, Operating Rooms; Desinfección de las Manos, Quirófanos, and Boolean connectors AND/OR in the period between 2006 and 2016. Results: Five guidelines of surgical hand preparation and two regulations on product evaluation for surgical hand preparation were consulted. Twenty-two articles were identified in the database search and seven were selected: four literature reviews - three of them were systematic reviews - and three studies evaluating cost and ecological sustainability. Conclusion: In the last decades, there have been major changes in the type of antiseptic product, which favored the use of alcoholic preparation (AP), without using water and brush, considering the cost-effectiveness and ecological sustainability when compared to traditional procedures such as surgical hand scrubbing with Polyvinylpyrrolidone Iodine (PVP-I) or Chlorhexedine Gluconate (CHG). To incorporate best practices based on scientific evidence, a programmatic approach must be adopted, policies, and programs must be implemented in order to manage including products and processes and monitor compliance with the procedures.

Keywords: Hand disinfection. Operating rooms. Hand hygiene.

RESUMEN: Objetivo: Describir tendencias y desafíos en la preparación quirúrgica de las manos. Método: Revisión de literatura narrativa, consulta a los manuales internacionales y nacionales actuales, además de consulta a Cochrane Database of Systematic Reviews, al portal BVS, y a la base de datos LILACS, IBECS, MEDLINE, Nursing Reference Center y SciELO, utilizando los descriptores: Desinfecção das Mãos, Salas Cirúrgicas; Hand Disinfection, Operating Rooms; Desinfección de las Manos, Quirófanos y conectores booleanos AND/OR en el período entre 2006 y 2016. Resultados: Se consultaron cinco manuales sobre la preparación quirúrgica de

'Enfermeira; Especialista em Prevenção e Controle de Infecção; Doutora em Enfermagem pela Escola de Enfermagem da Universidade de São Paulo (EEUSP); Docente do curso de Mestrado Profissional em Enfermagem da Faculdade Israelita de Ciências da Saúde Albert Einstein - São Paulo (SP), Brasil. E-mail: julyaeka@gmail.com

Rua Ouvidor Peleja, 235, apto. 102 - Vila Mariana - CEP: 04128-000 - São Paulo (SP), Brasil.

Recebido: 01 set. 2016 - Aprovado: 27 set. 2016

DOl: $10.5327 / Z 1414-4425201600040008$ 
las manos y dos normatizaciones de evaluación de productos para la preparación quirúrgica de las manos. Veintidós fueron los artículos identificados en la búsqueda en la base de datos y se seleccionaron 7: cuatro revisiones de literatura — tres de ellas revisiones sistemáticas; y tres estudios evaluando el costo y la sustentabilidad ecológica. Conclusión: En las últimas décadas, hubo grandes cambios referente al tipo de producto antiséptico — favoreciendo el uso de preparación alcohólica (PA), sin el uso de agua y cepillo, representando costo-efectividad y sustentabilidad ecológica comparada a los procedimientos tradicionales como la degermación quirúrgica de las manos con polivinil-pirrolidona yodada (PVP-I) o Gluconato de Clorhexidina (CHG). Para incorporar mejores prácticas basadas en evidencias científicas, se debe adoptar un abordaje programático, implementar políticas y programas que rigen los procesos y productos utilizados, así como el control de ese cumplimiento. Palabras clave: Desinfección de las manos. Quirófanos. Higiene de las manos.

\section{INTRODUÇÃ̃O}

A segurança do paciente cirúrgico é considerada uma preocupação mundial que atinge os pacientes de países desenvolvidos e em desenvolvimento. A infecção relacionada à assistência à saúde (IRAS), e especialmente as infecções de sítio cirúrgico, constituem um problema de saúde pública pela magnitude e pelo impacto na morbi-mortalidade ${ }^{1}$.

Como parte da campanha Aliança Mundial para a Segurança do Paciente (World Alliance for Patient Safety), lançada em outubro de 2004, a Organização Mundial da Saúde (OMS) publicou, em junho de 2008, o manual "WHO Guidelines for Safe Surgery (First Edition)" para garantir uma assistência mais segura ao paciente cirúrgico, sendo que um de seus objetivos focava na seguinte premissa: "A equipe irá usar de maneira consistente métodos conhecidos para minimizar os riscos para infecção de sítio cirúrgico (ISC)².

Entre as medidas preventivas de IRAS do paciente cirúrgico, no período peri-operatório, está a higiene das mãos (HM). No ano de 2016, na campanha "Clean care is safer care", do dia 05 de maio, a OMS utilizou o tema: "Olhe suas mãos: a higiene das mãos promove o cuidado cirúrgico seguro", cujo cartaz encontra-se disponível na página da ANVISA ${ }^{3}$.

Tendo em vista a segurança do paciente cirúrgico, esta revisão de literatura teve a seguinte questão norteadora: quais são as tendências do preparo cirúrgico das mãos no cenário internacional e nacional e, por conseguinte, quais são os desafios?

\section{OBJETIVO}

Descrever as tendências e os desafios no preparo cirúrgico das mãos, por meio da revisão de literatura.

\section{MÉTODO}

Realizada revisão de literatura narrativa, por meio de consulta aos manuais internacionais e nacionais atuais, à Cochrane
Database of Systematic Reviews, e ao portal BVS, à base de dados LILACS, IBECS, MEDLINE, Nursing Reference Center e SciELO, utilizando os seguintes descritores (palavras-chave e Medical Subject Heading - MeSH): Desinfecção das Mãos, Salas Cirúrgicas; Hand Disinfection, Operating Rooms; Desinfección de las Manos, Quirófanos e conectores booleanos AND/OR no período entre 2006 e 2016.

Foi realizada a leitura dos resumos e selecionados artigos que abordassem as mudanças significativas ao longo do tempo no preparo cirúrgico das mãos quanto a produtos, técnica e tempo do procedimento, assim como análise de custo-efetividade.

\section{RESULTADOS}

Foram consultados cinco manuais sobre preparo cirúrgico das mãos e duas normatizações de avaliação de produtos para esta finalidade. Do total de 22 artigos identificados durante a busca, 7 foram incluídos: 4 revisões de literatura — sendo 3 revisões sistemáticas - e 3 estudos avaliando o custo e a sustentabilidade ecológica.

\section{Recomendações sobre o preparo cirúrgico das mãos}

Os seguintes manuais foram consultados: Prevenção de Infecção de Sítio Cirúrgico (Centers for Disease Control and Prevention$\mathrm{CDC})^{4}$, Higiene das mãos em Serviços de Saúde $(\mathrm{CDC})^{5}$, Higiene das Mãos em Serviços de Saúde (OMS) ${ }^{6}$, Manual da Association of periOperative Registered Nurses (AORN) ${ }^{7}$ e Manual de HM da Anvisa ${ }^{8}$.

No período perioperatório, existem basicamente dois componentes de $\mathrm{HM}^{48}$ :

1. higiene rotineira das mãos: friccionar as mãos com produto alcoólico — se não houver sujeira visível nelas ou lavar com sabonete contendo ou não antisséptico (higienização simples ou higienização antisséptica);

2. antissepsia pré-cirúrgica das mãos: corresponde ao preparo cirúrgico das mãos com produto degermante à 
base de Polivinilpirrolidona Iodo (PVP-I) ou Gluconato de Clorexidina (CHG), ou ainda fricção com preparação alcoólica (PA).

Os Centers for Disease Control and Prevention (CDC) - no manual de Prevenção de Infecção de Sítio Cirúrgico ${ }^{4}$ - traz como recomendação a degermação cirúrgica, por no mínimo dois a cinco minutos, das mãos e dos antebraços até os cotovelos. Somente em 2002, no manual de Higiene das Mãos em Serviços de Saúde, os $\mathrm{CDC}^{5}$ passam a recomendar, além da utilização de sabão antimicrobiano, o produto alcoólico para a antissepsia cirúrgica das mãos, com atividade residual, antes de calçar luvas estéreis para realizar procedimento cirúrgico. Recomendam seguir as instruções do fabricante ao utilizar produto alcoólico e lavar previamente as mãos e antebraços com sabão não-antimicrobiano e secar completamente antes de aplicar a PA. Após aplicar a PA como recomendado, as mãos e antebraços devem secar completamente antes de calçar luvas estéreis. Orientam, ainda, evitar o tempo de degermação excessivo (10 minutos) e o uso de escova - que não são necessários e podem contribuir para dermatites nas mãos e nos antebraços.

A OMS, em $2009^{6}$, publicou o manual de HM em consonância com as recomendações dos CDC (2002), reforçando que a antissepsia cirúrgica das mãos deve ser realizada utilizando antisséptico degermante ou PA apropriados, de preferência com um produto que assegure a atividade residual, antes de calçar as luvas estéreis. E, se as mãos estiverem visivelmente sujas, lavá-las com sabonete líquido normal antes do preparo cirúrgico das mãos, removendo a sujeira sob as unhas, usando um limpador de unhas, de preferência em água corrente. Caso a qualidade da água não seja assegurada no bloco operatório, recomenda-se a antissepsia cirúrgica das mãos com PA antes de calçar as luvas estéreis ao realizar procedimentos cirúrgicos.

A técnica para realizar a antissepsia cirúrgica das mãos usando antisséptico degermante à base de PVP-I ou CHG consta dos seguintes passos ${ }^{6}$ :

- friccionar todas as superfícies das mãos e os antebraços durante o tempo recomendado pelo fabricante geralmente de dois a cinco minutos —, não sendo necessários longos períodos de fricção - por exemplo, dez minutos - e contraindica o uso de escovas;

- enxaguar as mãos e antebraços em água corrente em uma única direção, das pontas dos dedos para o cotovelo;

- secar as mãos e antebraços com uma compressa estéril e técnica asséptica, antes de vestir avental e calçar luvas.
Na antissepsia cirúrgica das mãos utilizando PA, recomenda-se ${ }^{6}$ :

- utilizar PA para antissepsia cirúrgica das mãos com atividade prolongada (residual), seguindo as instruções do fabricante para os tempos de aplicação;

- aplicar o produto apenas em mãos secas, uma quantidade suficiente do produto para manter as mãos e os antebraços úmidos com o produto alcoólico durante todo o procedimento de preparação das mãos para a cirurgia;

- não combinar sequencialmente preparações degermantes e preparações alcoólicas;

- após aplicação da PA conforme recomendação, deixar secar rigorosamente mãos e antebraços antes de calçar luvas estéreis ${ }^{6}$.

Segundo a $\mathrm{AORN}^{7}$, o preparo cirúrgico das mãos deve ser realizado antes de calçar luvas estéreis para procedimentos cirúrgicos ou invasivos. Recomenda-se utilizar agente antimicrobiano destinado à antissepsia das mãos ou uma PA para friç̧ão das mãos com atividade persistente e cumulativa documentada que atenda aos requerimentos da Food and Drug Administration (FDA). O produto para a antissepsia cirúrgica das mãos a ser selecionado deve considerar a análise de efetividade do produto, dos requisitos de aplicação e a aceitação pelo usuário?.

O manual da Anvisa "Segurança do Paciente - Higienização das Mãos"8 recomenda o uso de escovas de cerdas macias e descartáveis no preparo cirúrgico das mãos apenas em leito ungueal e subungueal. A duração do procedimento deve ser de três a cinco minutos para a primeira cirurgia e de dois a três minutos para as cirurgias subsequentes. Quanto à técnica: recolher, com as mãos em concha, o antisséptico e espalhar nas mãos, no antebraço e no cotovelo. No caso de escova impregnada com antisséptico, pressionar a parte da esponja contra a pele e espalhar por todas as partes ou friccionar as mãos, os espaços interdigitais e os antebraços, mantendo as mãos acima dos cotovelos.

\section{Métodos de avaliação da eficácia antimicrobiana de produtos para o preparo cirúrgico das mãos}

Basicamente são dois os métodos de avaliação da eficácia antimicrobiana para aprovação de antissépticos para o preparo cirúrgico das mãos: EN 12791, da Comité Européen de Normalisation (CEN $)^{9}$, e, E 1115, da American Society for Testing and Methods $(\mathrm{ASTM})^{10}$. Esses testes verificam a redução da microbiota residente das mãos, além da persistência e/ ou efeito cumulativo.

A norma europeia EN $12791^{9}$ preconiza o teste em 18 a 22 sujeitos, cujas mãos são divididas (split-hands) para avaliar 
o efeito imediato em uma mão enquanto a outra continua enluvada para avaliar o efeito sustentado/residual em 3 horas. Utiliza-se o desenho de estudo tipo cruzado (cross-over), em que ocorrem dois experimentos, com intervalo de uma semana, para comparação de redução bacteriana. O produto-referência é o n-propanol $60 \%$ (por volume), aproximadamente três mililitros durante três minutos para manter as mãos úmidas e o produto-teste deve seguir as recomendações do fabricante, mas deve ser utilizado em tempo menor que cinco minutos. As amostras microbianas são colhidas após a lavagem das mãos com sabonete sem atividade antimicrobiana (valor basal), imediatamente após a antissepsia (efeito imediato) e após três horas com mãos enluvadas (efeito residual), pelo método de fricção das pontas dos dedos em placas com meio de cultura e neutralizantes, uma para cada mão. O produto será aprovado se:

- valores imediatos e três horas não podem ser menores que do produto referência (n-propanol 60\%);

- se o produto tiver atividade residual, a liberação bacteriana da pele deve ser menor que o produto referência na $3^{\mathrm{a}}$ hora.

A norma americana ASTM E $1115^{10}$ — in vivo - , avalia o efeito imediato e persistente. O desenho de estudo empregado é o de estudo randomizado, cego, grupos paralelos (parallel arm), cujo tamanho da amostra é definido segundo a fórmula $\mathrm{n} \geq 2 S^{2}(\mathrm{Za} / 2+Z \mathrm{~b})^{2} / \mathrm{D}^{2}$, sendo $S^{2}$ a variação estimada, $\mathrm{Za} / 2$ corresponde ao nível do teste - para 5\%, nível teste $=1,96$ $\mathrm{Zb}$ é o poder de teste - para $80 \%$, poder $=0,842$ - e e D é a diferença clínica significante das exclusões. O produto-teste é utilizado por cinco dias consecutivos: $1^{\circ}$ e $5^{\circ}$ dias: apenas uma antissepsia; $2^{\circ}, 3^{\circ}$ e $4^{\circ}$ dias: três vezes por dia, com um intervalo mínimo de uma hora entre os procedimentos; total de 11 procedimentos no final do estudo. As amostras microbianas foram colhidas: antes do início do estudo (baseline); imediatamente após a antissepsia (efeito imediato); três horas e seis horas após a antissepsia com mãos enluvadas no dia 1 (efeito residual) e nos dias 2 e 5 (efeito cumulativo), utilizando o método glove juice. Para que o produto seja aprovado, deve atender aos seguintes requisitos:

- dia 1: dentro de um minuto após o procedimento, reduzir $1-\log _{10}$; após seis horas, não exceder o baseline (efeito residual);

- dia 2: dentro de um minuto após a última aplicação do dia ( $3^{\circ}$ uso), reduzir $2-\log _{10}$;

- dia 5: dentro de um minuto após o procedimento, reduzir $3-\log _{10}$ (efeito cumulativo).

\section{Efetividade de preparação alcoólica no preparo cirúrgico das mãos}

Três revisões sistemáticas avaliaram a PA no preparo cirúrgico das mãos: Gonçalves et al. ${ }^{11}$, Tanner et al. ${ }^{12}$, e Liu et al. ${ }^{13}$.

A revisão sistemática de Gonçalves et al. ${ }^{11}$ teve como objetivo comparar a eficácia antimicrobiana de preparações alcoólicas com os produtos tradicionais (PT) na antissepsia cirúrgica das mãos. Foram avaliados 25 estudos. Na maioria, as PA tiveram uma redução microbiana maior ou igual aos PT, e as taxas de ISC foram similares em cinco estudos. Os autores concluíram que existem evidências científicas que suportam a segurança das PA para antissepsia cirúrgica das mãos.

A revisão de Tanner et al. ${ }^{12}$ teve como objetivo avaliar os efeitos de antissepsia cirúrgica das mãos na prevenção de ISC; o objetivo secundário foi avaliar o número de unidades formadoras de colônia (UFC) de bactérias nas mãos da equipe cirúrgica. Quatorze estudos foram incluídos na revisão atualizada de 2006. Quatro estudos relataram o resultado das taxas de ISC e não mostraram diferença entre PA e produtos degermantes, e dez estudos relataram número de UFC, mas não as taxas de ISC. Os autores, no entanto, concluem que os estudos em geral eram realizados com um número de amostra pequeno, e outros não apresentavam dados ou análises que pudessem ser interpretados ou relacionados com os resultados clínicos. Esses fatores reduziram a qualidade da evidência.

A revisão de Liu et al. ${ }^{13}$ avaliou o efeito de técnicas de preparo cirúrgico das mãos na integridade da pele e na incidência de ISC. Foram incluídos dez estudos, sendo oito estudos clínicos randomizados e dois estudos clínicos não randomizados. Comparando a PA com degermantes à base de CHG/PVP-I (escova/sem escova), não houve diferença nas taxas de ISC, porém a PA foi melhor tolerada e causou menos problemas de pele. A técnica sem escova teve melhor desempenho do que com escovação na condição da pele. Os autores concluem que o protocolo de preparo cirúrgico das mãos com PA pode ser tão eficaz quanto o protocolo que utiliza o preparo tradicional na prevenção de ISC.

Uma revisão de literatura de Widmer et al. ${ }^{14}$ abordando o estado da arte sobre o preparo cirúrgico das mãos, sintetizou as evidências e os principais objetivos desta preparação cirúrgica, bem como os critérios para a escolha dos produtos atualmente em uso. Entre as conclusões, contraindicam o uso de escovas para a degermação cirúrgica das mãos e fortalecem o uso de PA devido à ação antimicrobiana rápida, ao amplo espectro de atividade antimicrobiana, à menor ocorrência de efeitos colaterais e à ausência de risco de contaminação das mãos pela água. Recomendam lavar as mãos antes da 
antissepsia cirúrgica somente se estiverem visivelmente sujas, e que lavá-las com sabonete sem antimicrobiano quando a equipe cirúrgica entrar no ambiente cirúrgico é o suficiente.

Quanto à técnica do preparo das mãos com PA, durante todo o procedimento, as mãos devem permanecer molhadas com o álcool durante a fricção, exigindo, portanto, cerca de 9 a $15 \mathrm{~mL}$, dependendo do tamanho das mãos. O tempo necessário para friç̧ão da PA depende da formulação, com exposição de três minutos, mas pode ser reduzido para um minuto e meio ou menos para algumas formulações ${ }^{14}$.

Jehle et al. ${ }^{15}$ quantificaram o volume de água utilizado na degermação cirúrgica das mãos para estimar a economia de água e investigar o custo envolvido na adoção de PA no preparo cirúrgico das mãos, em um estudo prospectivo durante um ano. Considerando o período padrão de três minutos no procedimento de degermação cirúrgica das mãos, estimou-se o uso de água em 18,5 L. O total de água utilizada para 3,25 procedimentos por cirurgia foi $60,2 \mathrm{~L}$, que, multiplicado por 15.500 procedimentos cirúrgicos ao ano, evidenciou um consumo de água anual de 931.938 L na degermação cirúrgica. Quanto aos custos relativos, consideraram ser vantajoso utilizar PA em relação à PVP-I ou ao CHG, de acordo com o volume utilizado da PA (6 mL).

Tavolacci et al. ${ }^{16}$ compararam a eficácia da antissepsia cirúrgica com a PA versus degermação cirúrgica, e determinaram os custos de ambas as técnicas de preparo cirúrgico das mãos. Foi realizada uma revisão de literatura para comparação da eficácia no MEDLINE. Os custos foram estimados com base em custos hospitalares-padrão. A literatura mostrou que a PA tem uma eficácia antimicrobiana imediata semelhante à degermação cirúrgica, mas a PA teve um efeito mais duradouro. O uso de PA reduziu os custos em 67\%. Dessa forma, conclui-se que a PA é uma alternativa de baixo custo para o preparo cirúrgico das mãos.
Um estudo nacional realizado por Graf et al..$^{17}$ avaliou o custo -efetividade de técnica de antissepsia com a PA — duração de um minuto-versus a escovação com CHG sob a perspectiva hospitalar brasileira. O custo total com a PA foi $46 \%$ inferior ao custo médio da técnica com escova de $\mathrm{CHG}$. No cenário ecológico, a redução de 18,5 L de água por procedimento, quando realizada a técnica com PA, gera economia financeira, além do próprio recurso hídrico poupado e redução de descarte de resíduos como as escovas.

\section{CONCLUSÃO}

Os manuais e os estudos apontam para as vantagens e o custo-efetividade das PAs no preparo cirúrgico das mãos, tais como: menor tempo de procedimento - propiciando uma provável adesão ao mesmo - , melhor condição da pele das mãos, maior eficácia antibacteriana, redução de custos, economia de água e redução de resíduos sólidos.

Os desafios no preparo cirúrgico das mãos são:

1. necessidade de produção científica nacional para entender a nossa realidade e/ ou cultura quanto à aceitação e implementação da PA nos serviços brasileiros;

2. prover atualização científica aos profissionais de saúde, em especial às equipes cirúrgicas;

3. avaliar a adesão às medidas recomendadas, utilizando indicadores de estrutura, processos e resultados.

Por fim, para mudar a prática, recomenda-se envolver os setores e as equipes - multisetorial e multidisciplinar para implementar as melhores práticas baseadas em evidências científicas, elaborando um projeto de melhoria: avaliar a situação atual (medida basal), implementar as estratégias de melhoria, e, posteriormente, avaliar o impacto.

\section{REFERÊNCIAS}

1. Kirkland KB, Briggs JP, Trivette SL, Wilkinson WE, Sexton DJ. The impact of surgical-site infections in the 1990s: attributable mortality, excess length of hospitalization, and extra costs. Infect Control Hosp Epidemiol. 1999;20(11):725-30.

2. World Health Organization. World Alliance for Patient Safety. WHO Guidelines for Safe Surgery (First Edition). Genebra: World Health Organization; June 2008. p. 43-71.

3. Agência Nacional de Vigilância Sanitária (Ministério da Saúde). 5 de maio de 2016 - Dia Mundial de Higiene das Mãos! Disponível em: http://www20.anvisa.gov.br/segurancadopaciente/index.php/ noticias/89-5-de-maio-de-2016-dia-mundial-de-higiene-das-maos
4. Mangram AJ, Horan TC, Pearson ML, Silver LC, Jarvis WR, the Hospital Infection Control Practices Advisory Committee. Guideline for the prevention of surgical site infection, 1999. Infect Control Hosp Epidemiol 1999; 20(4):247-280 [acesso em ago. 2016]. Disponível em: http://www.cdc.gov/hicpac/pdf/ guidelines/SSI_1999.pdf

5. Boyce JM, Pittet D. Guideline for hand hygiene in health-care settings: recommendations of the Healthcare Infection Control Practices Advisory Committee and HICPAC/SHEA/APIC/IDSA Hand Hygiene Task Force. MMWR Morb Mortal Wkly Rep; 2002; 51:1-45. Disponível em: http://www.cdc.gov/mmwr/PDF/rr/ rr5116.pdf 
6. World Health Organization. WHO Guidelines on Hand Hygiene in Health Care: a Summary. Genebra: World Health Organization; 2009. [acesso em ago 2016]. Disponível em: http://whqlibdoc.who.int/ hq/2009/WHO_IER_PSP_2009.07_eng.pdf

7. AORN. Recommended practices for hand hygiene in the perioperative setting. In: Perioperative Standards and Recommended Practices. Denver, CO: AORN, Inc; 2013. p. 63-74.

8. Agência Nacional de Vigilância Sanitária (Ministério da Saúde). Segurança do Paciente - Higienização das mãos. Brasília, 2009.

9. ComitéEuropéen de Normalisation. EN 12791. Chemical disinfectants and antiseptics. Surgical hand disinfection. Test method and requirements. Brussels: European Committee for Standardization, 2005.

10. American Society for Testing and Methods. E1115. Department of Health and Human Services. Food and Drug Administration. Tentative final monograph for health-care antiseptic drug products; Proposed Rule. Fed Reg. 1994;59:31401-52.

11. Gonçalves KJ, Graziano KU, Kawagoe JY. Revisão sistemática sobre antissepsia cirúrgica das mãos com preparação alcoólica em comparação aos produtos tradicionais. Rev Esc Enferm USP. 2012;46(6):1484-93.
12. Tanner J, Dumville JC, Norman G, Fortnam M. Surgical hand antisepsis to reduce surgical site infection. Cochrane Database Syst Rev. 2016;(1).

13. Liu LQ, Mehigan S. The Effects of Surgical Hand Scrubbing Protocols on Skin Integrity and Surgical Site Infection Rates: A Systematic Review. AORN J. 2016;103(5):468-82.

14. Widmer AF, Rotter M, Voss A, Nthumba P, Allegranzi B, Boyce J, et al. Surgical hand preparation: state-of-the-art. J Hosp Infect. 2010;74(2):112-22.

15. Jehle K, Jarrett N, Matthews S. Clean and green: saving water in the operating theatre. Ann R Coll Surg Engl 2008; 90(1):22-4.

16. Tavolacci MP, Pitrou I, Merle V, Haghighat S, Thillard D, Czernichow P. Surgical hand rubbing compared with surgical hand scrubbing: comparison of efficacy and costs. J Hospital Infect. 2006;63:(1)55-9.

17. Graf ME, Machado A, Mensor LL, Zampieri D, Campos R, Faham L. Antissepsia cirúrgica das mãos com preparações alcoólicas: custoefetividade, adesão de profissionais e benefícios ecológicos no cenário de saúde. J Bras Econ Saúde. 2014;6(2):71-80. 